\title{
Multidimensional measurement by using 3-D PMD sensors
}

\author{
T. Ringbeck, T. Möller, and B. Hagebeuker \\ PMDTechnologies GmbH, Am Eichenhang 50, 57076 Siegen, Germany
}

\begin{abstract}
Optical Time-of-Flight measurement gives the possibility to enhance 2-D sensors by adding a third dimension using the PMD principle. Various applications in the automotive (e.g. pedestrian safety), industrial, robotics and multimedia fields require robust three-dimensional data (Schwarte et al., 2000). These applications, however, all have different requirements in terms of resolution, speed, distance and target characteristics. PMDTechnologies has developed 3-D sensors based on standard CMOS processes that can provide an optimized solution for a wide field of applications combined with high integration and cost-effective production. These sensors are realized in various layout formats from single pixel solutions for basic applications to low, middle and high resolution matrices for applications requiring more detailed data. Pixel pitches ranging from 10 micrometer up to a 300 micrometer or larger can be realized and give the opportunity to optimize the sensor chip depending on the application.
\end{abstract}

One aspect of all optical sensors based on a time-of-flight principle is the necessity of handling background illumination. This can be achieved by various techniques, such as optical filters and active circuits on chip. The sensors' usage of the in-pixel so-called SBI-circuitry (suppression of background illumination) makes it even possible to overcome the effects of bright ambient light. This paper focuses on this technical requirement. In Sect. 2 we will roughly describe the basic operation principle of PMD sensors. The technical challenges related to the system characteristics of an active optical ranging technique are described in Sect. 3, technical solutions and measurement results are then presented in Sect. 4. We finish this work with an overview of actual PMD sensors and their key parameters (Sect. 5) and some concluding remarks in Sect. 6.

Correspondence to: B. Hagebeuker

(b.hagebeuker@PMDTec.com)

\section{Basic principle}

\subsection{Time-Of-Flight basic principle}

As the Time-Of-Flight principle has already been described in detail in many technical publications (Kraft et al., 2004; Ringbeck et al., 2003; Buxbaum et al., 2001), only the basic principles will be discussed here.

Figure 1 shows the basic Time-Of-Flight principle. In its most simple form, a light pulse is transmitted by a sender unit and the target distance is measured by determining the turnaround time the pulse needs to travel from the sender to the target and back to the receiver. With knowledge of the speed of light the distance can then easily be calculated. However, the receiver needs to measure with picosecond-accuracy the delay between start and stop, if millimeter-precision is required. To realize such system solutions with discrete components, as is done in today's TOF rangefinders, each component in the signal chain must have a very high system bandwidth.

In contrast Figs. 1 and 2 show the basic working principle of a PMD based range imaging camera. Rather than using a single laser beam (which would have to be scanned over the scene to obtain 3-D) the entire scene is illuminated with modulated light. The advantage of PMD devices is that we can observe this illuminated scene with an intelligent pixel array, where each pixel can individually measure the turnaround time of the modulated light. Typically this is done by using continuous modulation and measuring the phase delay in each pixel. In addition to this robust method of obtaining 3-D without scanning, the realization of the phase measurement in a quasi optical domain offers huge advantages compared to the above mentioned discrete solutions. This is one reason, why we do not require an optical reference channel for most applications.

Published by Copernicus Publications on behalf of the URSI Landesausschuss in der Bundesrepublik Deutschland e.V. 


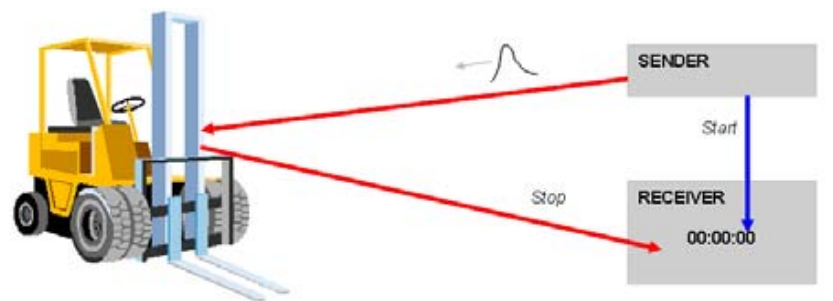

Fig. 1. Time-Of-Flight measurement principle with pulsed light.

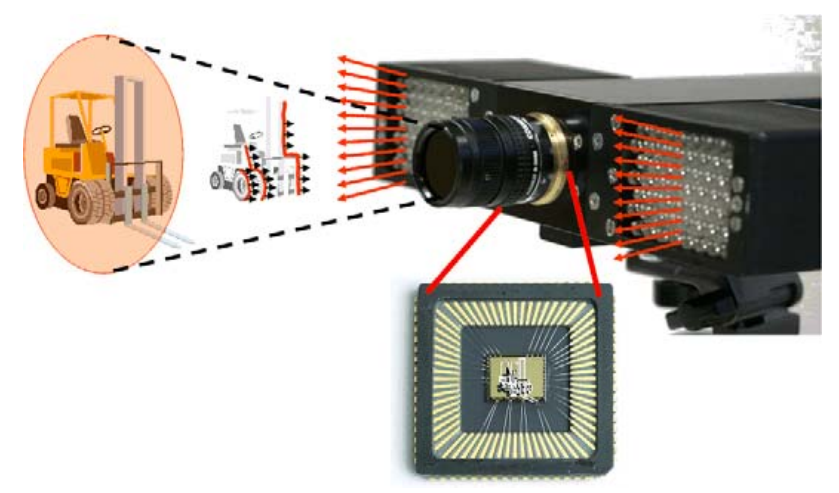

Fig. 2. Imaging Time-Of-Flight, measurement based on PMD.

\subsection{Operation principle of PMD}

This section describes the principle of a simplified PMD sensor realized in CMOS technology. Because the complete mixing process of the electric and optical signal takes place within each pixel we call the PMD elements "smart pixels". Figure 3a shows an illustration of a single pixel PMD sensor element. It is a five-terminal device with an optical input window, i.e. two transparent modulation electrodes in the middle of the illustration. These light sensitive Photogates are isolated from the substrate by a thin oxide layer. The gates are conductive and transparent for the received light. On the left and the right there are readout diodes which are connected to the pixel readout circuitry. In a PMD pixel the movement of generated charge carriers can be controlled by the reference signal applied to the modulation gates. This way one can influence a charge transport to the left or to the right side. The potential distribution in the surface region is influenced by these push-pull voltages leading to a "dynamic seesaw" for the generated charge carriers (Kraft et al., 2004).

If the incident light is constant and the modulation is a rectangular signal with a duty cycle of $50 \%$ the generated charge carriers within a modulation period move to the left and to the right equally. At the end of such a modulation process the output voltages at the readout nodes are the same as those shown in Fig. 3b.

If, however, the incident light is modulated (e.g. also as a rectangular signal), and there is no phase (phase $=0^{\circ}$ ) delay between modulation of light and detector, then all charge car-
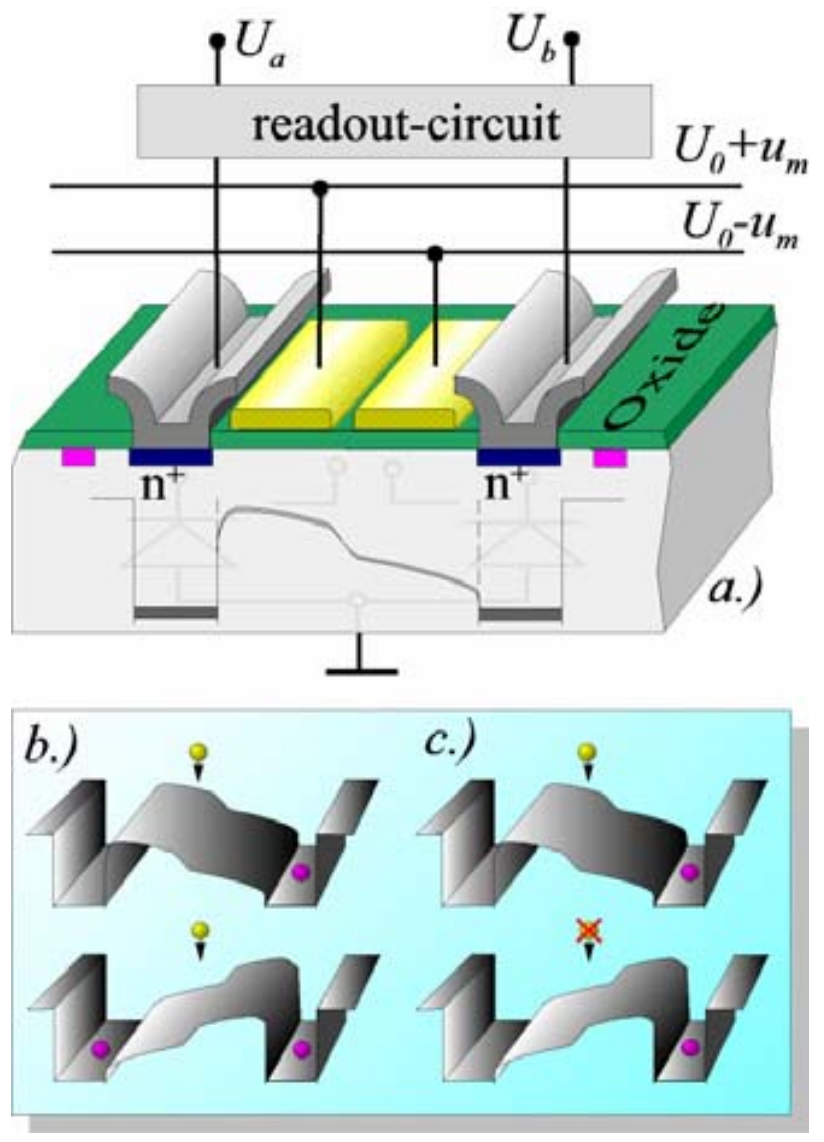

Fig. 3. Operation principle of PG-PMD.

riers will be moved to one of both readout diodes (Fig. 3c). For other phase delays, the modulation of the light and its phase delay compared to the electrical reference signal results in a difference between the two output voltages. This difference of both output nodes is directly dependent on the phase delay between light and pixel modulation. This data can be used to calculate the distance from a light-reflecting object to the sensor as described in a later section.

Figure 4 gives an impression of how both readout nodes will behave for different target distances.

\subsection{Analysis of the ACF (autocorrelation function)}

To calculate the distance between target and camera, the autocorrelation function of electrical and optical signal is analyzed by a phase-shift algorithm (Fig. 5). Using four samples $A_{1}, A_{2}, A_{3}$ and $A_{4}$ - each shifted by 90 degrees - the phase, which is proportional to the distance, can be calculated using the following equation.

$\varphi=\arctan \left(\frac{A_{1}-A_{3}}{A_{2}-A_{4}}\right)$ 

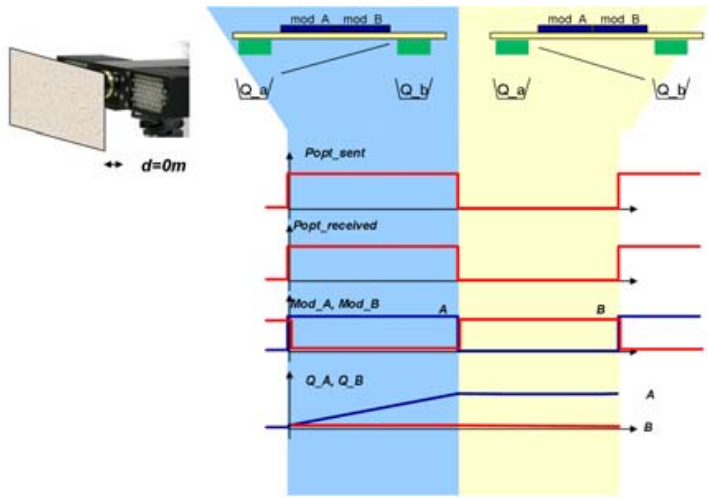

a) Target distance: $\mathbf{0 ~} \mathbf{m}$

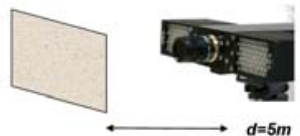

c) Target distance: $5 \mathbf{m}$

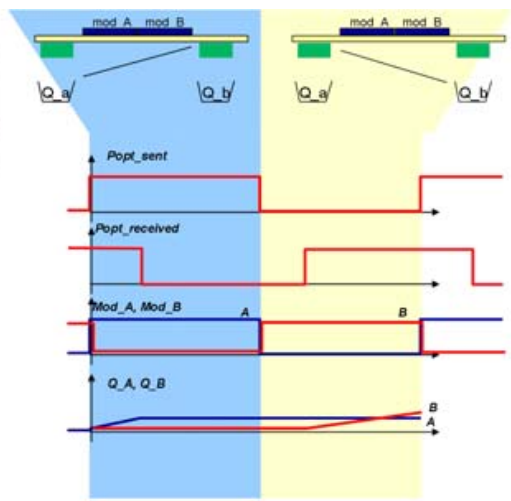

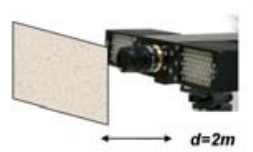

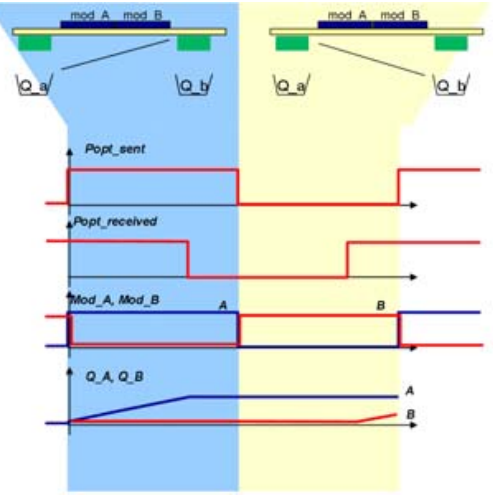

b) Target distance: $2 \mathrm{~m}$

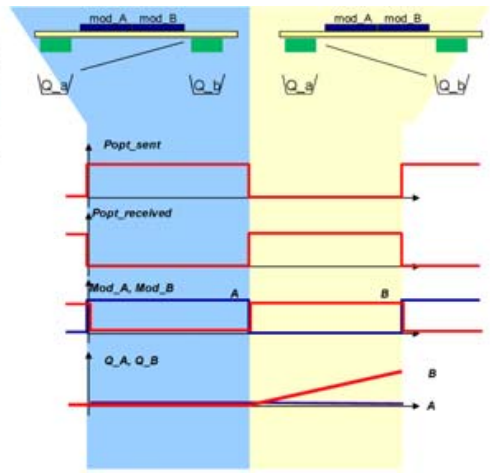

c) Target distance: $7.5 \mathrm{~m}$

Fig. 4. PMD operation with different target distances.

Signal Phase $\varphi$ : distance

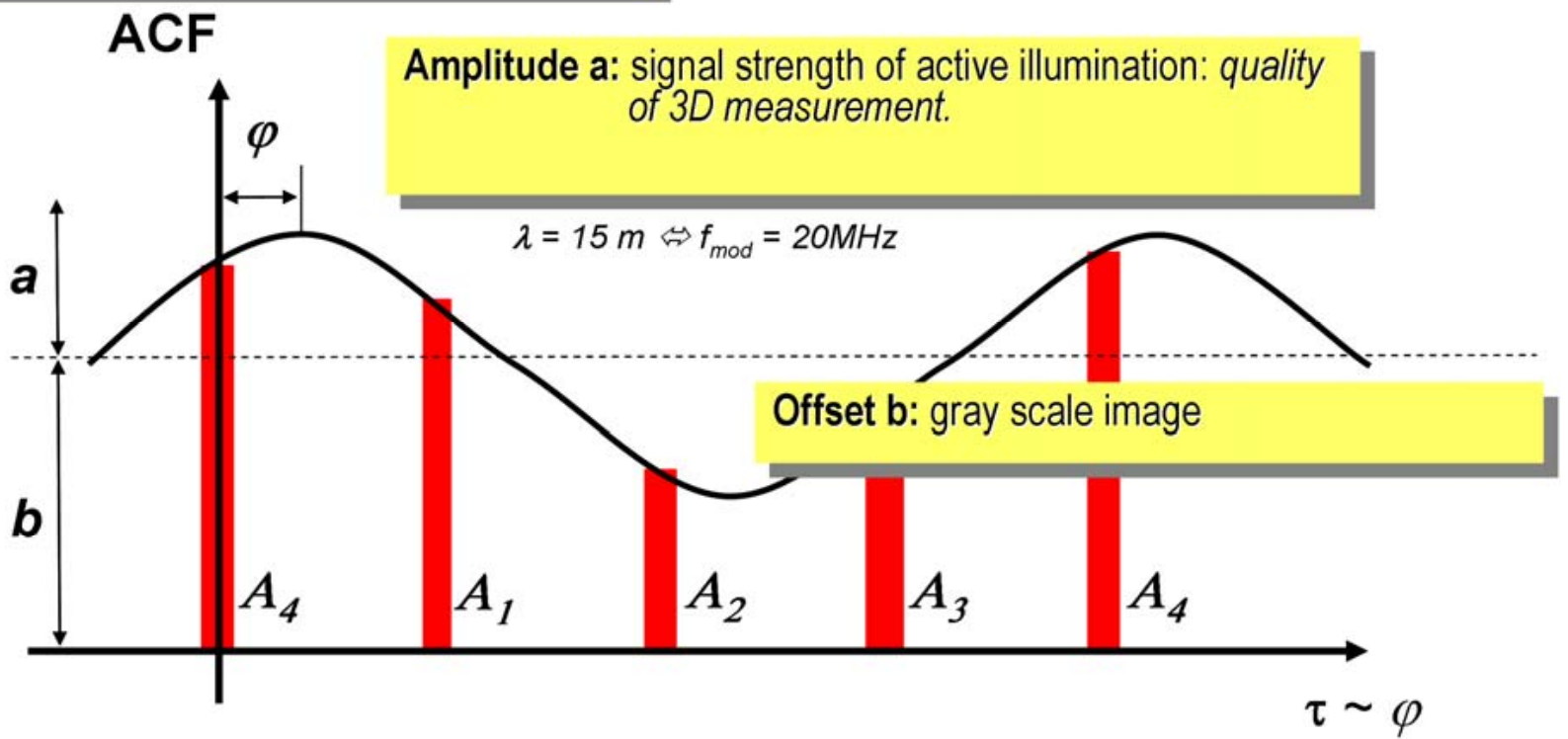

Fig. 5. ACF (autocorrelation function), Signal Phase, Amplitude and Offset. 


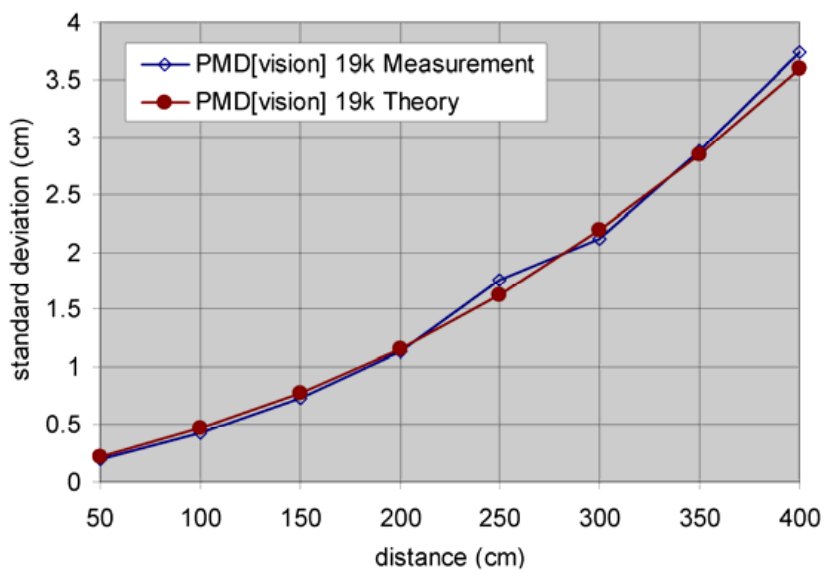

Fig. 6. Calculated standard deviation versus measurement results.

In addition to the phase-shift of the signal, two other values can be extracted. At first, the signal strength of the received signal (amplitude):

$a=\frac{\sqrt{\left(A_{1}-A_{3}\right)^{2}+\left(A_{2}-A_{4}\right)^{2}}}{2}$

And the second value, the offset $b$ of the samples, represents the gray-scale value of each pixel.

$b=\frac{\sum_{1}+\sum_{2}+\sum_{3}+\sum_{4}}{4}$

The distance $d$ to the target is given by Eq. (4). At a modulation frequency of $f_{\text {mod }}=20 \mathrm{MHz}$, for example, the wavelength $\lambda_{\text {mod }}$ is $15 \mathrm{~m}$. The maximum distance for the target is $d_{\max }=\lambda_{\bmod } / 2$ because the active illumination has to cover the distance twice: from the sender to the target and back to the sensor chip.

$d=\frac{c \cdot \varphi}{4 \pi \cdot f_{\bmod }}$

\section{Challenges}

2.1 Technical requirements: how much light for accurate measurement?

It is obvious that for an active optical measurement system the reliability is directly influenced by the amount of (active) light which arrives at the sensor. In Lange (2000) and Buxbaum (2002) the dependency of statistical measurement uncertainty as a function of optical signal strength and system noise is derived. The measurement uncertainty $\mathrm{dR}$ of a PMD time of flight system can be calculated as:

$d R=\frac{1}{\sqrt{N_{\text {phase }}}} \cdot \frac{1}{k_{\text {tot }} \cdot \frac{S}{N}} \cdot \frac{\lambda_{\text {mod }}}{\sqrt{8} \cdot \pi}$

Where $k_{\text {tot }}$ is the modulation contrast, $S$ is the number of (active) signal electrons, $N$ is the equivalent number of noise electrons (including shot noise of active light, background light and dark current as well as thermal noise, reset noise, system noise and ADC noise), $N_{\text {phase }}$ is the number of measurements and $\lambda_{\text {mod }}$ is the wavelength of the modulation signal in meters. An alternative notation of this product of $k_{\text {tot }}^{\prime}$ and $S^{\prime}$ is fully equivalent, where $S^{\prime}$ represents the complete number of integrated electrons, including background light and dark current and $k_{\text {tot }}^{\prime}$ represents the overall system contrast, also including dark current and background light offset. Please note that for both cases the product of $k_{\mathrm{tot}}$ and $S$ represents the "active signal share" after integration (demodulation amplitude).

The helpful thing is that for each measurement, all parameters of the above equation are known. That means, that each single measurement more or less directly offers the information of its own reliability, without requiring the use of statistics. Subsequently, thresholds can be set to ignore measurement results with great uncertainty.

One can also easily see from the equation above, what modifications of the system and sensor parameters can be made to improve the measurement accuracy. The following section describes the influence of the parameters mentioned above.

High PMD contrast and high modulation contrast of the light sources improves the distance accuracy. Optimizing the contrast doesn't influence the signal-to-noise ratio but rather decreases the inaccuracy by a factor of $1 / k_{\text {tot }}$. The maximum value of $k_{\text {PMD }}$ and $k_{\text {light }}$ is 1 .

$k_{\mathrm{tot}}=k_{\mathrm{PMD}} \cdot k_{\text {light }}$

It is always desirable to lower the total noise without affecting the signal. This can be done by reducing the dark current noise. Therefore special opto-CMOS processes with low dark current should be used. Especially in automotive applications where the temperature can be up to $125^{\circ} \mathrm{C}$, darkcurrent-optimized CMOS processes are essential for satisfactory performance because dark current doubles with every $8^{\circ} \mathrm{C}$. Improvements are also achieved by low amplifier- and reset-noise on-chip and low system-noise, as well as decreasing background illumination noise. However, since background illumination is an environmental parameter and not a system parameter, it is difficult to decrease the background illumination in an observed scene. The number of photons reaching the sensor can be reduced though. This is done by proper band pass filtering of the incoming light.

Increasing the signal by using stronger light sources increases the accuracy linearly if the photon shot noise of the uncorrelated light or other noise sources are dominant rather than the photon shot noise of the active illumination. Otherwise, if the photon shot noise $N_{\text {active }}$ of the active illumination is the most dominant part of the total noise $\mathrm{N}$, the distance accuracy increases by the square root of the signal.

$\frac{S}{N} \propto\left\{\begin{array}{ll}\sqrt{S}, & \text { if } N_{\text {active }}>N \\ S, & \text { else }\end{array}\right\}$ 


\begin{tabular}{|lc|}
\hline LED & \\
LED & $0.3 \mathrm{~nm} / \mathrm{K}$ \\
Temp. Automotive min & $-40{ }^{\circ} \mathrm{C}$ \\
Temp. Automotive max & $120^{\circ} \mathrm{C}$ \\
Temp. Automotive range & $160 \mathrm{~K}$ \\
& $48 \mathrm{~nm}$ \\
LED & $50 \mathrm{~nm}$ \\
Filter & $98 \mathrm{~nm}$ \\
& \\
Viewing angle & $20 \mathrm{deg}$ (diagonal) \\
Range & $40 \mathrm{~m}$ \\
Image Format & $1: 1$ \\
edge of image (object space) & $10.0 \mathrm{~m}$ \\
area of image (object space) & $99 \mathrm{~m} \mathrm{~m}^{\wedge} 2$ \\
\#LEDS & $100 \mathrm{LEDs}$ \\
optical power per LED & $0.04 \mathrm{~W} / \mathrm{LED}$ \\
total optical power of LEDs (CW) & $4 \mathrm{~W}$ \\
power density in object space (CW) & $0.040 \mathrm{~W} / \mathrm{m}^{\wedge} 2$ \\
power density in object space (CW, filtered) & $0.039 \mathrm{~W} / \mathrm{m}^{\wedge} 2$ \\
Burst-Operation & $5 \mathrm{Factor}$ \\
& $20 \mathrm{~W}$ \\
power desnisty in object space (Burst) & $0.20 \mathrm{~W} / \mathrm{m}^{\wedge} 2$ \\
power desnisty in object space (Burst, filtered) & $0.19 \mathrm{~W} / \mathrm{m}^{\wedge} 2$ \\
\hline
\end{tabular}

Fig. 7. Calculation sunlight vs. active light.

It must be kept in mind, that using more optical power comes at a price, and the overall costs of a TOF camera system can significantly rise by adding more LED or lasers. To avoid this, burst operation can be implemented. In burst operation, the light sources have the same average power and therefore the same number of light emitting elements, but higher peak power than in continuous operation. The shorter exposure time leads to the same number of signal electrons but less noise electrons due to less accumulated background light electrons. Thus, burst operation is effective under strong background light conditions, such as outdoor applications with sunlight. Another advantage of burst operation is the ability of detecting fast moving objects.

Large lens apertures lead to more photo generated electrons, both information holding electrons from the light source and unwanted ones from background light. In most cases the accuracy can be improved linearly with aperture enlargement since the number of photo generated electrons is a square function of the aperture. In very special cases, when the noise floor due to dark current, quantization etc is dominant, the improvement of accuracy increases with the square of the aperture.

Sensitivity of the PMD sensor can be enhanced by high fill factor and micro lenses. Good reflectivity and close targets will increase the signal, too. However, this is strongly dependent on the application and therefore not an essential part of design.

Another powerful method to improve the measurement accuracy is to use higher modulation frequencies (lower modulation wavelength $\lambda_{\text {mod }}$ ). For example doubling the frequency doubles the measurement accuracy. Physical limitations are the bandwidth of the light source and the bandwidth of the sensor. Furthermore the contrast may decrease at high frequencies, which will lower the effectiveness of this method.

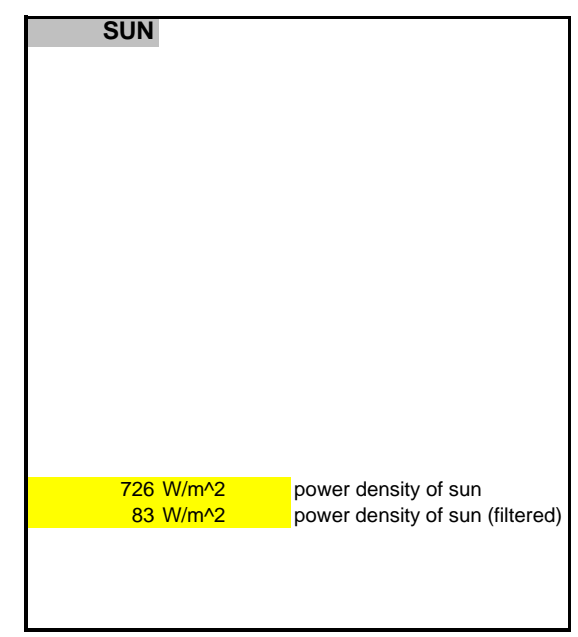

Figure 6 shows the comparison between the calculated accuracy and the measured one. For the calculation, the parameters described above were identified for a $160 \times 120$ pixel camera system. The measurement was done under well defined environment conditions. A good match of both curves can be observed. Hence, the performance of new applications can be easily estimated.

We can summarize that high modulation contrast of PMD and light source, optimized optical filters, high modulation frequency, good fill factor, large pixels and low noise floor are important design goals for PMD sensors and systems.

\subsection{Influence of sunlight}

For most outdoor applications sunlight is much brighter than the active illumination. This has two negative impacts on the TOF-system:

- Shot noise is increased (results in distance noise)

- A huge amount of the dynamics of the sensor is occupied by the sunlight (the sensor may saturate)

Figure 7 gives an overview of the calculation of the optical power of 100 LEDs in comparison to the sun for a typical application scenario. In this example the ratio between sun and active light is $18000=85 \mathrm{db}$ at a distance of $40 \mathrm{~m}\left(20^{\circ}\right.$ viewing angle). In the following section we will show how this ratio can be improved by intelligent system design. Firstly, the optical input signal is spectrally filtered. Although LEDs in the near infrared have a typical spectral width of $50 \mathrm{~nm}$, thermal drift of LEDs and automotive temperature range requirements must also be taken into account. These facts allow only a medium spectral filter width of approximately $100 \mathrm{~nm}$ to be used. In this case, the power density of the sun on the sensor is lowered and the ratio sun/active light goes down to 


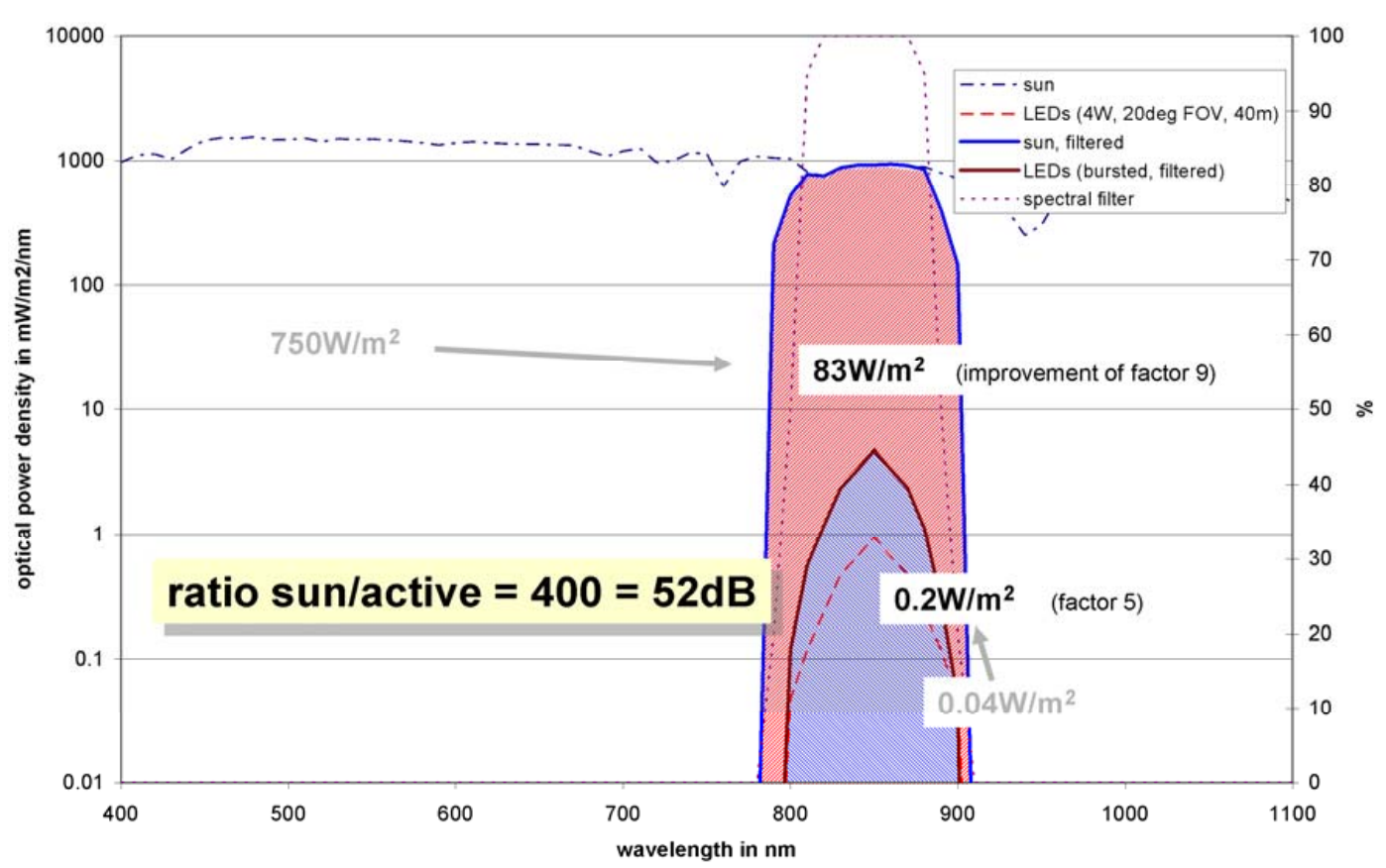

Fig. 8. Optical spectrum of sunlight with spectral filtering and active light in burst-mode.

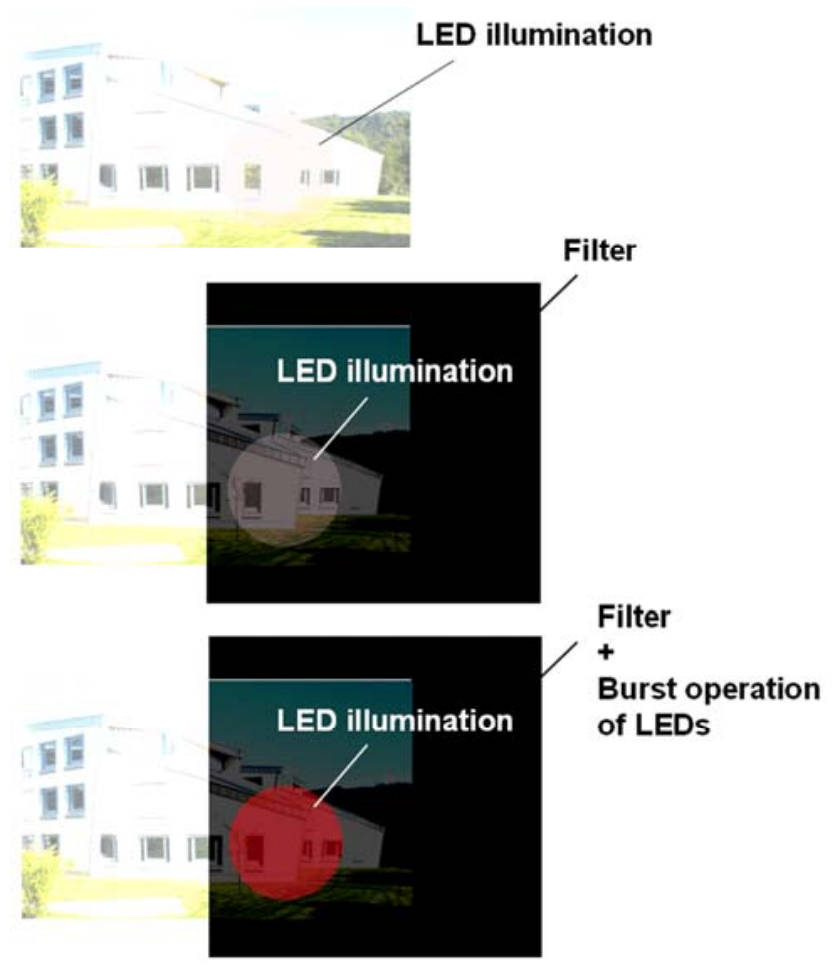

Fig. 9. Usage of optical filters and burst mode.

$2000=66 \mathrm{db}$. Further improvements can be achieved by using a burst mode for the LEDs. A burst operation of factor 5 results in a sunlight/active-light ratio of $400=52 \mathrm{db}$.
Figure 8 shows the result of both enhancements in a diagram and Fig. 9 shows the effects on an example picture. Spectral filtering and burst operation of the light source are effective methods to improve the "optical" SNR. The reasonable sunlight rejection of these introduced methods is about $-33 \mathrm{db}$. But the remaining ratio of sunlight/active signal is still poor: Even the filtered sun is still a factor of 400 brighter than the pulsed LEDs. Additional sunlight rejection methods have to be found.

The key challenges for optical TOF measurement are sensors with a high bandwidth and high sensitivity and a system with high dynamics to achieve sunlight robustness.

\section{Technical solutions}

\subsection{Definition of contrast}

Considering the output channels of the PMD sensor, two values are of importance: the difference of the readout channels delivering the phase information, and the sum of both channels which is proportional to the incident light and the overall photo generated charge carriers. As mentioned above, an important figure of merit for distance measurement is the ratio of the measured signal difference compared to the signal sum, the PMD contrast $k_{\mathrm{PMD}}$.

$k_{\mathrm{PMD}}=\frac{\left|Q_{A}-Q_{B}\right|}{Q_{A}+Q_{B}}=\frac{\left|\Delta V_{A}-\Delta V_{B}\right|}{\Delta V_{A}+\Delta V_{B}}$

The contrast has its maximum value when using phase shifts of $0^{\circ}$ and $180^{\circ}$ in terms of electrical and optical signal. 


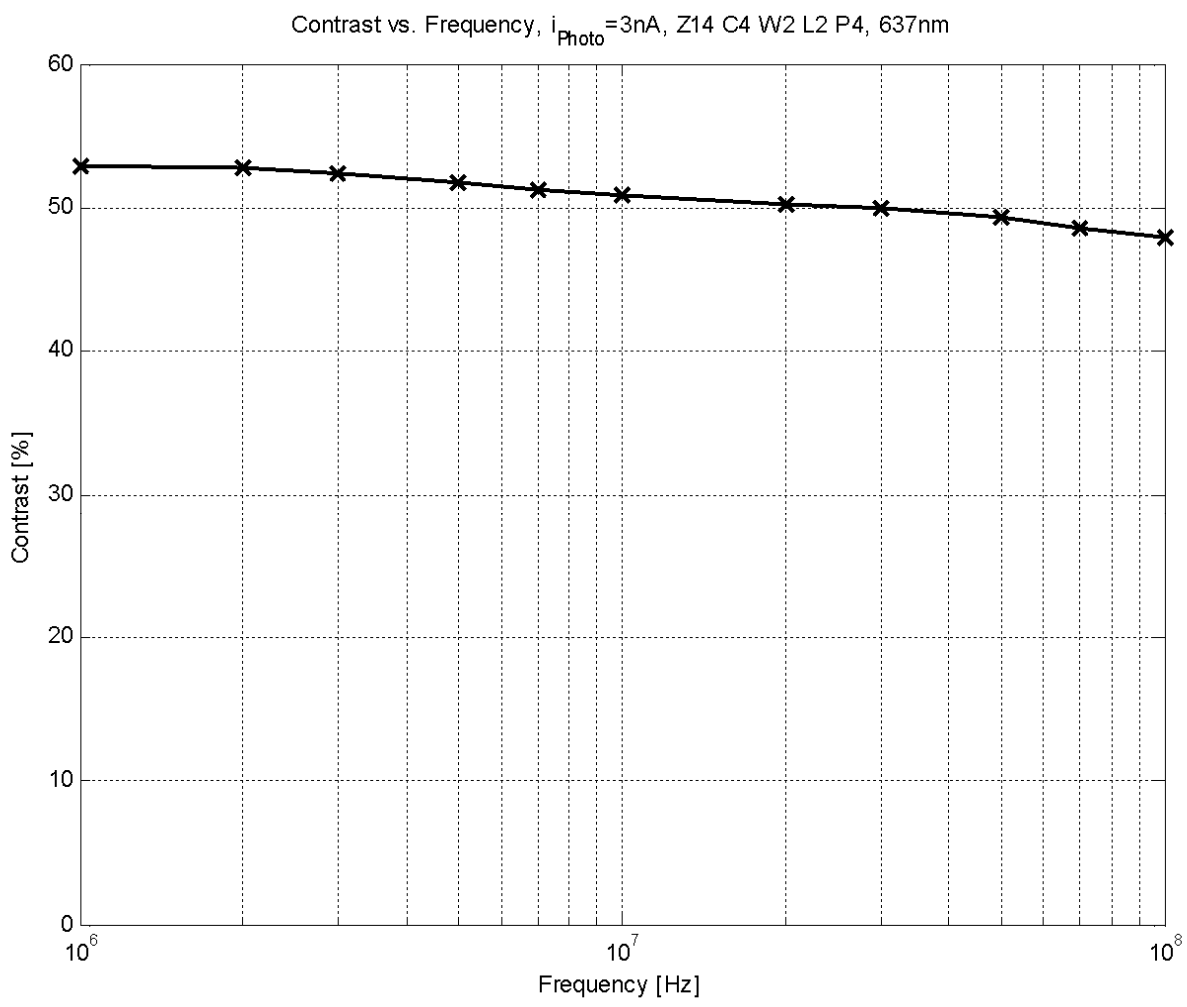

Fig. 10. Exemplary contrast behaviour showing high contrasts combined with high bandwidth.

Depending on modulation waveform, an ideal device can have a maximum contrast of $100 \%$, that is, all photo generated charge carriers contribute to the phase measurement since they are properly distributed to the corresponding readout channels (perfect device and system). Therefore, the contrast reflects the separation ability of the device in terms of charge carriers generated by correlated light. By definition the contrast is significantly affected by the signal sum, therefore determination of the device contrast is only valid without any uncorrelated illumination, and so the measurement must be carried out with a modulated light signal only.

The contrast of a PMD device is mainly dependent on pixel architecture and geometry. The main focus during pixel design is always the active region of the device which is defined by the light sensitive area and the light sensitive modulation gates. Of course, other requirements must also be met, such as overall pixel fill factor and photoresponsivity. Our development has led to PMD pixels with scalable pixel pitch without a loss of performance. The active pixel size can be modified based on application requirements from $10 \mu \mathrm{m} \times 10 \mu \mathrm{m}$ up to $300 \mu \mathrm{m} \times 300 \mu \mathrm{m}$ or even larger. This feature is based upon a special kind of pixel layout - PMD pixels with large dimensions can therefore offer the same performance as small designs. The pixels are optimized for high bandwidth (beyond $100 \mathrm{MHz}$ ) to increase pixel performance and accuracy. Figure 10 shows exemplary contrast behaviour of a typical 2-gate PMD pixel. The contrast of 3gate pixels is typically higher $(60 \%)$. It can be seen that a high contrast can be attained over several decades of modulation frequency which is mandatory for accurate distance measurements (Eq. 5).

\subsection{SBI - Suppression of background illumination}

The mentioned optical filtering and bursting of light sources might not be sufficient to reduce disturbing uncorrelated sunlight. The remaining background illumination can still saturate the sensor for typical integration times which can lead to a decrease of accuracy or pixel failures. In addition, there are other causes for uncorrelated disturbances. The unavoidable dark current of a semiconductor device especially, can have a considerable influence particularly at higher temperatures. For example, an increase in temperature of 80 degrees leads to a dark current which can be 1000 times higher and thus would significantly decrease measurement accuracy. Obviously solutions must be found, in order to reduce the disturbing influence of any uncorrelated signal.

The possibility to combine the sensor with on-chip application-specific circuitry is fundamental for an enhanced smart optical sensor. To improve the dynamic range of the PMD device, additional circuitry for the instantaneous suppression of background illumination (SBI) has been introduced. Using such a special readout technique allows precise 


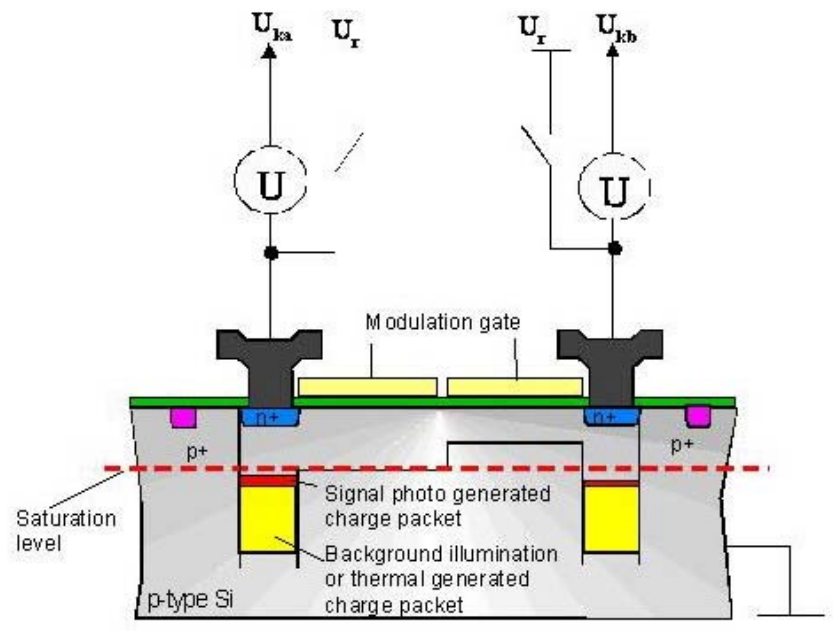

no SBI

Fig. 11. Working Principle of PMDTec's patented SBI circuitry.
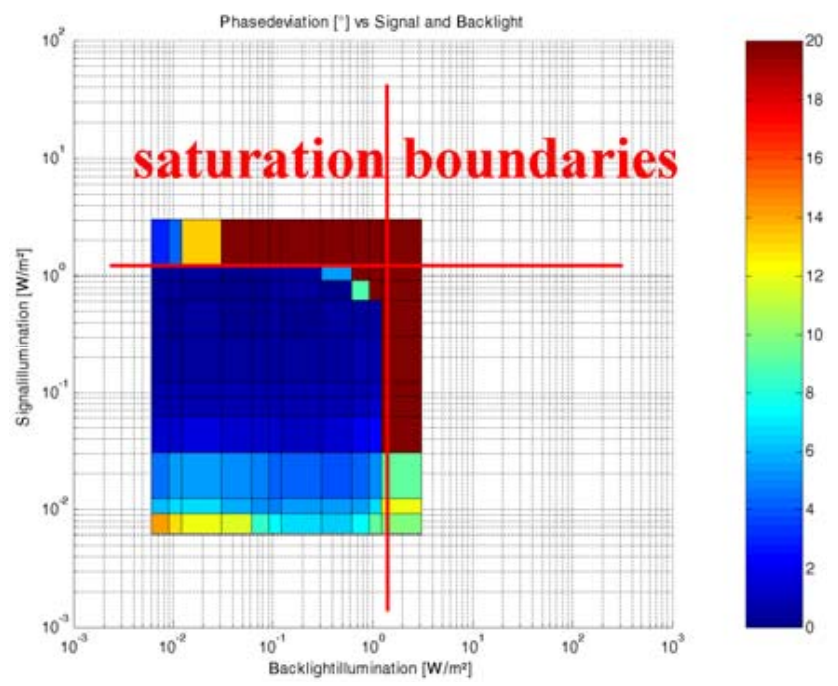

Fig. 12. Dynamic Measurements (SP, no SBI).

3-D measurements even in environments with huge uncorrelated signals at high temperatures. Therefore this principle is a key feature for a number of range imaging applications where the existence of disturbing uncorrelated signals is unavoidable. The working principle of PMDs patented SBI circuitry is illustrated in the following figure:

To eliminate uncorrelated signals which are distributed on both readout sides equally, additional compensation sources have been added. These sources inject additional charges in both readout terminals in order to instantaneously compensate for the saturation effects of uncorrelated signals during the integration process. This compensation means that the dynamic range of the following readout stage can be com-

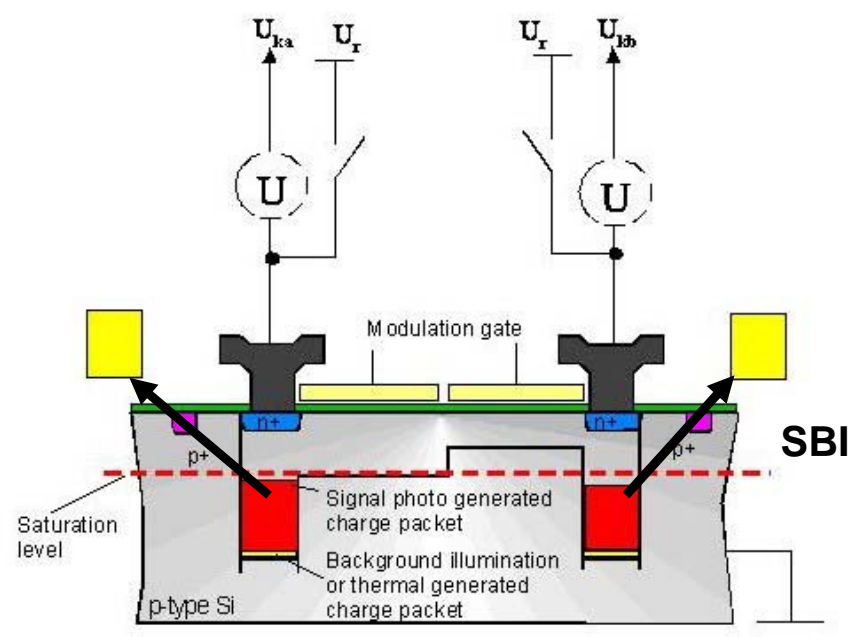

SBI

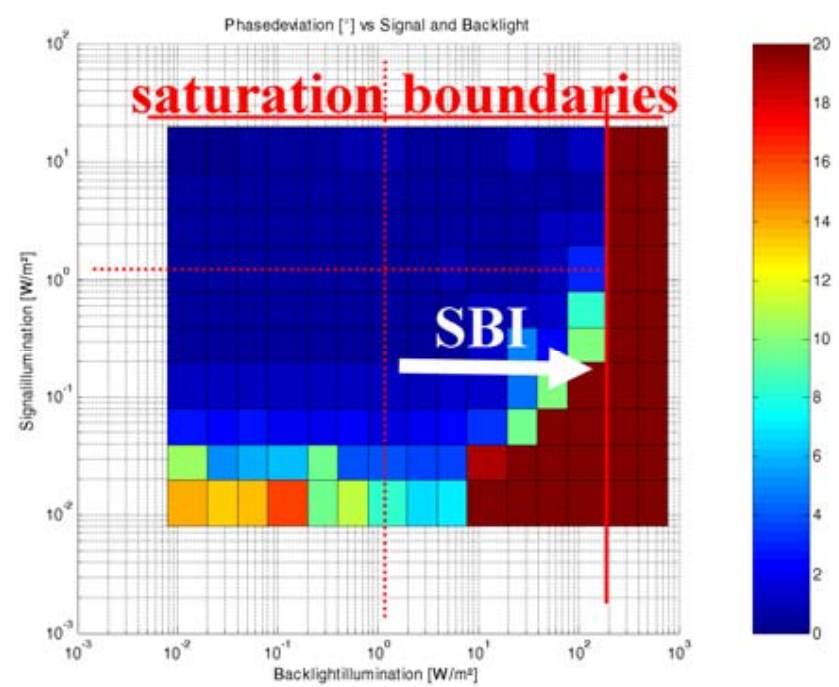

Fig. 13. Dynamic Measurements (SP, SBI).

pletely used by the correlated signal information only. In other words, the readout circuitry of the pixel ideally sees only the correlated signal and no unwanted background DC component. To offer a wide range of applications and flexibility this concept has been implemented in most of our PGPMD arrays (Fig. 16). An active SBI circuitry will reduce the background DC component, and so the scenes ambient light greyscale information will be lost. Alternatively a similar greyscale image can also be extracted out of the acquired amplitude data (active light greyscale image), even with active SBI. Also the SBI feature can be electronically deactivated or adjusted so that it is always possible to acquire both high-quality 3-D measurement and 2-D greyscale information, depending on the application needs. 


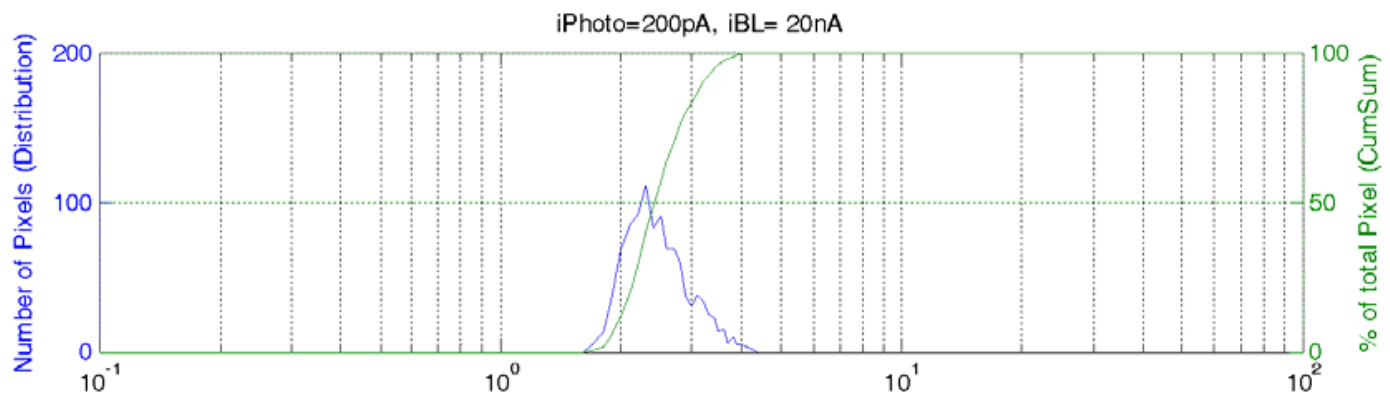

Fig. 14. Statistic analysis $(64 \times 16$ Matrix, SBI).

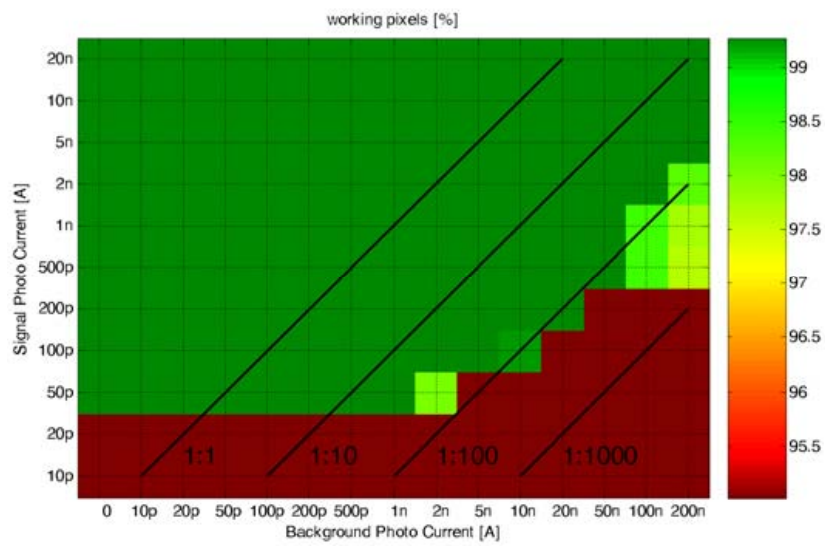

Fig. 15. Dynamic range $(64 \times 16$ Matrix, SBI).

\subsection{Measurements}

To measure the functionality of PMD sensors with SBI circuitry, a phase measurement procedure must be used. This is due to the fact that the total number of collected and stored electrons is modified by the usage of an active SBI, and so the standard definition of contrast (Eq. 8) can no longer be used as characteristic device performance parameter. To examine the achieved dynamic range (with regard to tolerable signal and background intensity levels), a real (Time-Of-Flight) phase measurement using a 4-phase algorithm is performed under controlled conditions (Eq. 9).

$$
\begin{aligned}
& \varphi_{\mathrm{TOF}}=\arctan \left(-\frac{\sum_{i} u_{\mathrm{Int}}\left(\varphi_{i}\right) \cdot \sin \left(\varphi_{i}\right)}{\sum_{i} u_{\mathrm{Int}}\left(\varphi_{i}\right) \cdot \cos \left(\varphi_{i}\right)}\right) \\
& \varphi_{i} \quad=0, \frac{1}{2} \pi, \pi, \frac{3}{2} \pi
\end{aligned}
$$

Using an automated measuring station, the intensities of signal and background light required for measurement can be adjusted very reproducibly in our optical laboratory. To verify reliable phase measurement using subsequent statistic analysis, a high number of measurements are made for several combinations of signal and background intensity.

Figure 12 shows the resulting standard deviation as a function of signal and background intensity for a single PMD
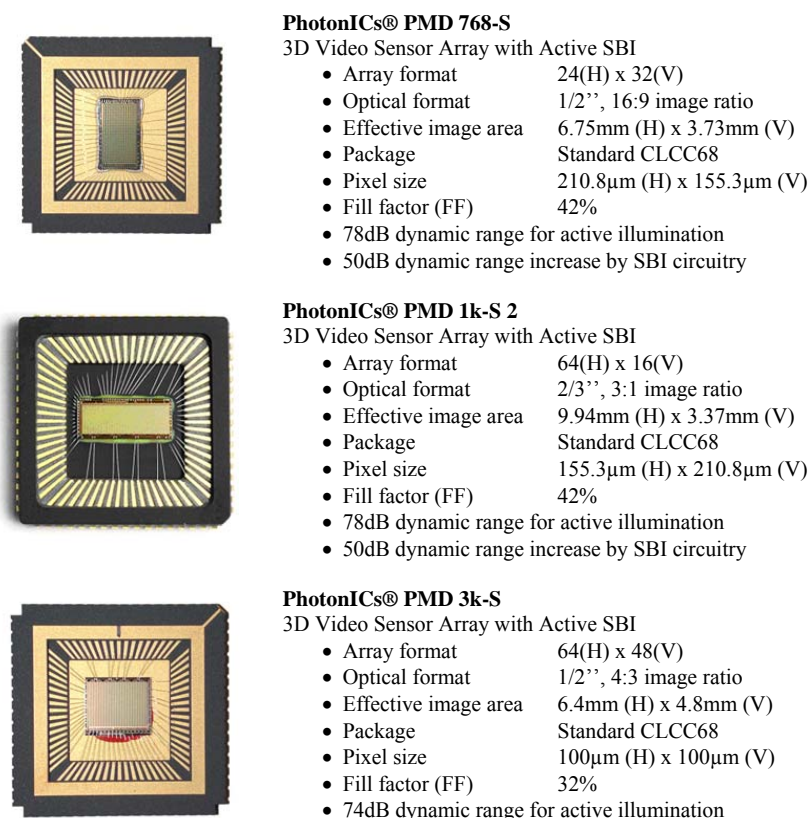

PhotonICs ${ }^{\circledR}$ PMD 3k-S

3D Video Sensor Array with Active SBI

- Array format 64(H) x 48(V)

- Optical format $1 / 2$ ", 4:3 image ratio

- Effective image area $6.4 \mathrm{~mm}(\mathrm{H}) \times 4.8 \mathrm{~mm}(\mathrm{~V})$

- Package Standard CLCC68

- Pixel size $\quad 100 \mu \mathrm{m}(\mathrm{H}) \times 100 \mu \mathrm{m}(\mathrm{V})$

- Fill factor (FF) $\quad 32 \%$

- $74 \mathrm{~dB}$ dynamic range for active illumination

- $50 \mathrm{~dB}$ dynamic range increase by SBI circuitry

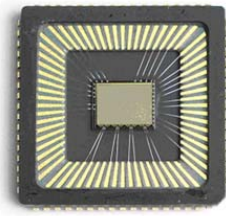

PhotonICs® PMD 19k

High Resolution 3D Video Sensor Array

- Array format $160(\mathrm{H}) \times 120(\mathrm{~V})$

- Optical format $\quad 1 / 2$ ', 4:3 image rati

- Effective image area $6.4 \mathrm{~mm}(\mathrm{H}) \times 4.8 \mathrm{~mm}(\mathrm{~V})$

- Package Standard CLCC68

- Pixel size $\quad 40 \mu \mathrm{m}(\mathrm{H}) \times 40 \mu \mathrm{m}(\mathrm{V})$

- Fill factor (FF) $\quad 31 \%$

- $65 \mathrm{~dB}$ dynamic range for active illumination

Fig. 16. PMD Sensor-Chips based on Photogate Technology.

pixel with disabled SBI. The overlaid saturation boundaries indicate the achieved dynamic range, limited by saturation of integration capacitors. In contrast, Fig. 13 shows the increased dynamic range with enabled SBI circuitry. The dynamic range of a typical pixel can be increased by a factor of 300 - that is, $50 \mathrm{~dB}$. Since quality of SBI performance may vary from pixel to pixel due to technical fabrication tolerances, each single pixel may deliver a slightly different dynamic range. Please note, that there are always pixels in the array with far higher SBI performance (factor 1000 and even 


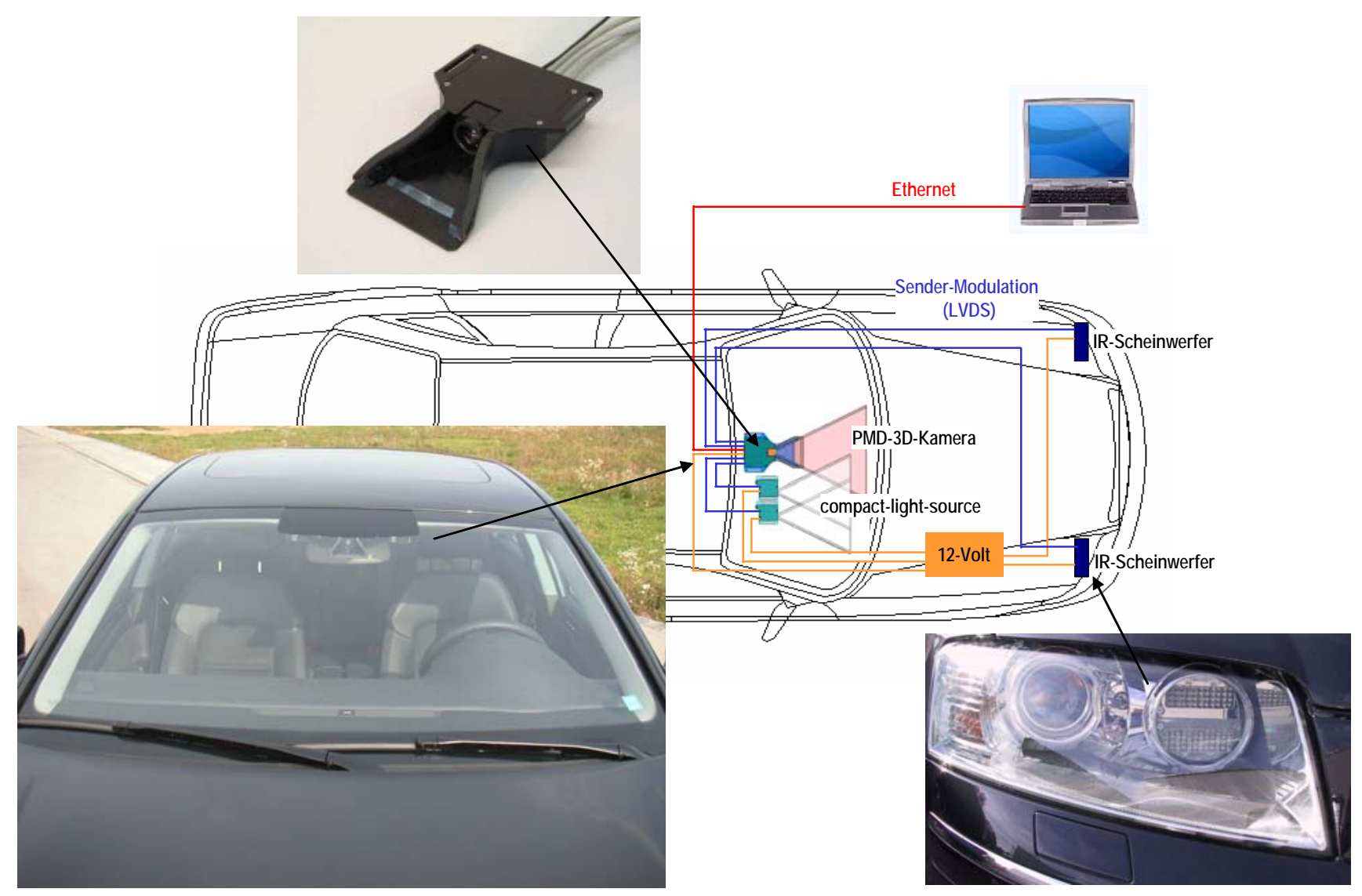

Fig. 17. Test car from 2005 with an A-Muster PMD camera system for pedestrian safety.

more). Therefore, in order to yield a more complete result, measurement should be extended from single pixel characterisation to parallel characterisation of all pixels of a PMD array with SBI circuitry.

The diagram in Fig. 14 shows the distribution of phase value standard deviation for a complete PMD array (sensor PhotonICs 1kS2) for fixed signal and background illumination levels. Statistical evaluation indicates that, $100 \%$ of the pixels achieve a phase accuracy of less than 4 degrees for a signal photo current of $200 \mathrm{pA}$, a background photo current of $20 \mathrm{nA}$ and an integration time of $1 \mathrm{~ms}$.

Figure 15 illustrates the SBI array performance for all tested intensities, for a given modulation frequency of $10 \mathrm{MHz}$, an integration time of $1 \mathrm{~ms}$ and a standard deviation of $5^{\circ}$, which corresponds to a distance resolution of $20 \mathrm{~cm}$. Combinations of intensities that result in at least $99.3 \%$ working pixels ( $5 \sigma$-range) are marked in green. That means for $99.3 \%$ of all pixels, the standard deviation is less than $5^{\circ}$. Combinations that result in less than $95 \%$ working pixels ( $3 \sigma$-range) are coloured red. To facilitate measurement results, ledger lines of constant background-to-signal ratio are overlaid. As can be seen, a background-to-signal ratio up to a factor of 400 , that is $52 \mathrm{db}$, is achieved for more than $95 \%$ of all pixels. Please consider, that the measurement certainty can be further increased by statistics and intelligent filter algorithms. These measurement results are based on an integration time of $250 \mu \mathrm{s}$.

\section{Technical implementation}

\subsection{Realized PMD Sensors}

For indoor and outdoor applications with different requirements, PMDTechnologies has developed sensors with specific features, like resolution, optical format and SBI. A short overview of four sensors with optical and mechanical dimension is given in the following figure. The sensors can be ordered for evaluation purposes.

All of these PMD Sensors are implemented in camera prototypes which shows the basic function principle or furthermore they are implemented in camera products like the PhotonICs ${ }^{\circledR}$ PMD 3k-S. In the next chapter, a camera system for pedestrian safety is introduced, which based on the PMD Sensor PhotonICs ${ }^{\circledR}$ PMD 1k-S. 


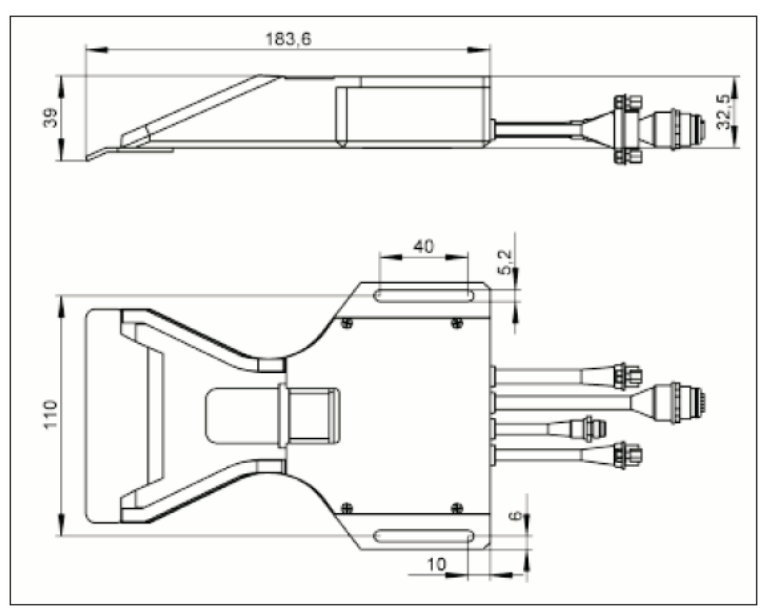

\begin{tabular}{|l|l|}
\hline Measurement Range & $10 \mathrm{~m} / 35 \mathrm{~m}$ \\
\hline Field of view & $\begin{array}{l}52^{\circ} \text { vertical } \\
18^{\circ} \text { horizontal }\end{array}$ \\
\hline DataUpdate Rate & $10,4 \mathrm{~ms}(96 \mathrm{~Hz})$ \\
\hline Distance Resolution per Pixel & $\pm 10 \mathrm{~cm}$ \\
\hline Operation Current of camera module & Max. $600 \mathrm{~mA}$ \\
\hline Temperature Range & $-10^{\circ}$ to $85^{\circ} \mathrm{C}$ \\
& $\left(-40^{\circ}\right.$ to $105^{\circ}$ in 2007$)$ \\
\hline Operating Voltage & 11 bis $18 \mathrm{DC}$ \\
\hline
\end{tabular}

Fig. 18. Technical Specification of A-Muster.

\subsection{Pedestrian safety camera system}

In Fig. 17, a camera system for pedestrian safety is depicted. This A-Muster camera is designed to demonstrate solutions for several front view application like Stop\&Go, PreCrash and Pedestrian Saftey therefore additional IRillumination sources are implemented inside the head lights to increase transmitted power and mearsurement range respectively. Other design spaces for illumination are determined and tested until beginning of 2007.

An actual sensor is available with the specification depicted in Fig. 18. The measurement range depends on illumination source. A range of $10 \mathrm{~m}$ is realized by an Illumination Source with $1 \mathrm{~W}$ optical power next to the camera system. A measurement range of above $35 \mathrm{~m}$ could be realized with illumination source in front of the car (i.g. inside the head lights) with and optical power more than $8 \mathrm{~W}$.

\section{Conclusion}

Time-Of-Flight systems based on the PMD-principle give the possibility of fast 3-D measurement with customizable resolutions depending on the application. High bandwidth and high sensitivity of the PMD pixels are the fundamentals for enhanced predictable measurement accuracy. Many applications (like outdoor) require robust operation even under very bright ambient light conditions. Spectral filtering combined with an active illumination in burst-mode improve the signal to noise ratio and are effective methods to reduce the impact of ambient light. However, for typical outdoor applications, additional sunlight rejection methods are necessary. PMDTechnologies has realized an outstanding ambient light rejection method, the active SBI circuitry realized as compact in-pixel electronics. This SBI circuitry removes all uncorre- lated signals instantaneously during the integration process. It is approved in the 6th sensor generation and works very reliable. The combination of all the presented techniques provides robust 3-D measurement under full sunlight conditions.

\section{References}

Buxbaum, B.: Optische Laufzeitentfernungsmessung und CDMA auf Basis der PMD-Technologie mittels phasenvariabler PNModulation, Ph.D. dissertation, University of Siegen, Germany, 2002.

Buxbaum, B. and Hagebeuker, B.: Dreidimensionale UmfeldErfassung, Elektronik Automotive, Nr. 5, pp. 77, Sep., 2005.

Buxbaum, B., Schwarte, R., Ringbeck, T., Grothof, M., and Luan, X.: MSM-PMD as correlation receiver in a new 3D-ranging system, SPIE - Remote Sensing, Laser Radar Techniques: Ranging and Atmospheric Lidar, Toulouse, 2001.

Kraft, H., Frey, J., Moeller, T., Albrecht, M., Grothof, M., Schink, B., and Hess, H.: Universität Siegen, B. Buxbaum, PMDTechnologies GmbH Siegen, 3D-Camera of High 3D-Frame Rate, Depth-Resolution and Background Light Elimination Based on Improved PMD (Photonic Mixer Device)-Technologies, OPTO 2004, AMA Fachverband, Nürnberg, 2004.

Lange, R.: 3D Time-of-flight distance measurement with custom solid-state image sensors in CMOS/CCD technology, Ph.D. dissertation, University of Siegen, Germany, 2000.

Lange, R., Seitz, P., Schwarte, R., et al.: Time-of-flight range imaging with a custom solid state image sensor, Proceedings of the SPIE, vol. 3823, pp. 180-191, Munich, 1999.

Ringbeck, T., Albrecht, M., Frey, J., Grothof, M., Heß, H., Kraft, H., Möller, T., Mosen, J., and Schink, B.: Time-of-Flight 3Dcamera for autonomous navigation and industrial automation, Nürnberg, Sensor, 2003.

Schwarte, R., Xu Z., Heinol H., et al.: A new active 3D-Vision system based on rf-modulation interferometry of incoherent light, 
SPIE - Intelligent Robots and Computer Vision XIV, vol. 2588, p. 126-134, Philadelphia, 1995.

Schwarte, R., Buxbaum, B., Heinol, H., Xu, Z., Schulte, J., Riedel, H., Steiner, R., Scherer, M., Schneider, B., and Ringbeck, T.: New Powerful Sensory Tool in Automotive Safety Systems Based on PMD-Technology, AMAA - Advanced Microsystems for Automotive Applications 2000, Berlin, 2000.
Spirig, T., Marley, M., Seitz, P., et al.: The multitap lock-in CCD with offset subtraction, IEEE Transactions on electron devices, vol. 44, no. 10, pp. 1643-1647, 1997. 